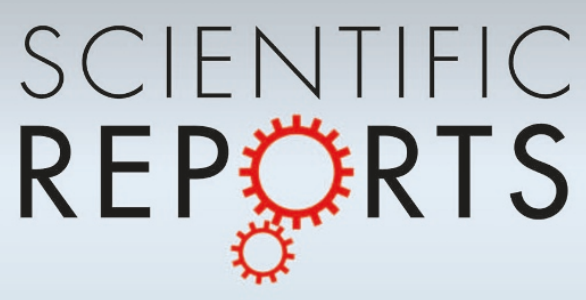

OPEN

SUBJECT AREAS:

NANOPARTICLES

MOLECULAR SELF-ASSEMBLY

STRUCTURAL PROPERTIES

SYNTHESIS AND PROCESSING

Received

3 April 2013

Accepted

19 August 2013

Published

10 September 2013

Correspondence and requests for materials should be addressed to

S.A. (camsa2@iacs. res.in) or S.S. (surajit@ tifrh.res.in)

* These authors contributed equally to this work.

† Current address: Tata Institute of Fundamental

Research, Centre for Interdisciplinary

Sciences, 21

Brundavan Colony,

Narsingi, Hyderabad 500075, India

\section{Origin of chains of Au-PbS Nano-Dumbbells in space}

\author{
Chandana Mondal*, Ali Hossain Khan*, Bidisa Das, Somobrata Acharya \& Surajit Senguptał
}

Centre for Advanced Materials, Indian Association for the Cultivation of Science, 2A \& 2B Raja S.C. Mullick Road, Jadavpur, Kolkata 700032, India.

Self-assembled, one-dimensional (1D) nanomaterials are amenable building blocks for bottom-up nanofabrication processes. A current shortcoming in the self-assembly of $1 \mathrm{D}$ nanomaterials in solution phase is the need for specific linkers or templates under very precise conditions to achieve a handful of systems. Here we report on the origin of a novel self-assembly of $1 \mathrm{D}$ dumbbells consisting of Au tipped $\mathrm{PbS}$ nanorods into stable chains in solution without any linkers or templates. A realistic multi-particle model suggests that the mesophase comprises 1D dumbbells arrayed in chains formed by anisotropic van der Waals type interactions. We demonstrate an alternative recognition mechanism for directing the assembly of the 1D dumbbells, based on effective interaction between the neighboring dumbbells consisting of Au tips with complementary crystallographic facets that guides the entire assembly in space.

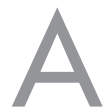
primary challenge in material science is the development of ordered architectures based on nanoscale building blocks in three dimensional (3D) solution phase ${ }^{1}$. Self-assembly is the main paradigm to achieve this goal since natural systems such as living cells mimic countless examples of functional self-assemblies in $3 \mathrm{D}$ space ${ }^{1}$. Previous reports on the self-assembly of nanomaterials primarily focused on the processes such as solvent evaporation ${ }^{2-4}$, molecular cross-linking ${ }^{5,6}$, template patterning ${ }^{7,8}$, dipolar interactions ${ }^{9-11}$, interdigitation of surfactant molecules ${ }^{12,13}$, or DNA as linkers ${ }^{14,15}$. The self-assembly of 1D nanorods is rather limited till date, there exists only a few reports using linker molecules ${ }^{16,17}$, or $\mathrm{DNA}^{18}$ utilizing the specificity to bind at certain faces. Key to these self-assembling routes is the requirement of a linker or guiding template that links the nanomaterials and defines designated positions within an assembly. Alternatively, there exists no procedure to reliably fabricate a long range periodic self-assembled array of nanorod chains in 3D liquid phase without using linkers or templates. Understanding the critical parameters controlling the stability of a spontaneous assembly of $1 \mathrm{D}$ nanorods is a necessary step for rationale control of materials processing conditions. Attempt towards modeling the system at equilibrium is extremely complex because direct experimental observations based on which theoretical analyses could be built and validated are lacking. Metal tipped nanorods/nanowires are the potential candidates for modular self-assembly using metal tips as natural contact points, recognition of element for directed self-assembly and holds promise for new strategies in self-assembly ${ }^{19,20}$. While making electrical contacts on a single nanorod for device fabrication is challenging, the metal-tipped nanostructures offers natural contact points for single nanocrystal based devices and may solve the problem of contacting nanorods to the external world $^{21,22}$. In addition, metal tipped nanorods are useful candidates for photocatalysis and biomedical applications $^{23-25}$. Here, we demonstrate a system of Au-tipped PbS rods (dumbbells) which spontaneously assemble as arrays at a mesoscopic scale in solution. We show, using high resolution transmission electron microscopy (HRTEM) and solution phase small angle X-ray scattering (SAXS) studies that the self-assembly in solution occurs without the support of any linker or template molecules. Using the experimental structural information and from ab-initio electronic structure calculations, we constructed a realistic atomistic model for the assembly using an anisotropic "patchy" potential ${ }^{26}$ which mimic the strongly orientation dependent interactions of the tips of dumbbells. Molecular dynamics simulations of this model allows to understand the parameters controlling the origin of free standing chains of dumbbells in solution and to predict future self-assembly strategies in 3D space. Understanding the mechanism of spontaneous assembly at mesoscopic scale is a key issue that might find wider applications in device fabrication processing from the solution phase.

\section{Results}

We synthesized the dumbbells in two steps; the first step involved preparation of PbS nanorods of $1.7 \mathrm{~nm}$ diameter and $\sim 12 \mathrm{~nm}$ in length by decomposition of $\mathrm{Pb}$-hexaethylxanthate in trioctylamine (TOA) ${ }^{27,28}$. In a 
second step, $\mathrm{PbS}$ nanorod suspension in toluene was stirred with $\mathrm{HAuCl}_{4}$ in methanol for 90 minutes at ice bath for metal deposition at the tips forming the dumbbells ${ }^{28}$. The dark field TEM image of the dumbbells dispersed from liquid phase shows that the dumbbells are assembled in stable arrays or chains in the solution phase (Fig. 1a). The dumbbells are self-assembled into arrays of chains typically exceeding $\sim 100 \mathrm{~nm}$ in length. Each chain consists of nearly $18-20$ dumbbells with their long axes preferentially leaning in a near parallel orientation with the adjacent one. Note that, $\mathrm{PbS}$ rods without Au tips forms disordered aggregates suggesting that chain formation is crucially linked to the presence of the Au tips (Supplementary Fig. $\mathrm{S} 1)^{27}$. A lateral periodicity of $3.5 \pm 0.2 \mathrm{~nm}$ (centre to centre distance $\sim 5.2 \mathrm{~nm}$ ) between adjacent dumbbells is evidenced along the chain lengths from the statistical profile analysis (Fig. 1b). The upright length of two TOA molecules $(2.24 \mathrm{~nm}$, geometrically calculated from the stretched chains) is smaller than the observed lateral distance of $3.5 \mathrm{~nm}$ indicating that the self-assembly is not mediated by the interdigitation of the TOA alkyl tails stretched normal to the long axes of the dumbbells (Supplementary Fig. S2). In order to confirm that the observed periodicity pre-exists in the liquid phase, we have performed SAXS of the dumbbells in toluene suspension (Fig. 1c) which shows that a sharp and intense peak appears at $2.212^{\circ}(2 \theta)$ followed by $2^{\text {nd }}$ and $3^{\text {rd }}$ order peaks at $4.427^{\circ}$ and $6.642^{\circ}$ respectively. The fundamental peak corresponds to a periodic spacing of $\sim 4 \mathrm{~nm}$ suggesting pre-existence of ordered assembly in solution phase.

The TEM and SAXS data unequivocally show that the observed periodicity along the chain length is larger than the length of the interdigitated layers of ligand molecules ${ }^{12,13}$. This implies that the self-assembly of dumbbells in spontaneous chains substantially differs from other reported assembly strategies relying on electrostatic, hydrophobic/hydrophilic interactions, template patterning or using linker molecules ${ }^{7,8,16,17,29,30}$. The HRTEM image (Fig. 1d) of the dumbbells reveals the rods with interplanar spacing of $0.29 \pm 0.02 \mathrm{~nm}$ corresponding to (200) d-spacing of the PbS bulk rocksalt structure. Selected area electron diffraction pattern also reveals $\mathrm{Au}$ fcc and $\mathrm{PbS}$ rock-salt phases of the dumbbells (Supplementary Fig. S3). Further, $\mathrm{Au}$ at the tips shows lattice spacings corresponding to traces of two different crystallographic facets namely (111) and (200) planes of the Au fcc structure (Figs. 1, d-f and Supplementary Figs. S4 and S5). Additionally, the Au tips at either ends of the dumbbells also have alternate crystallographic orientations along the length of the chain (Fig. $1 \mathrm{~g}$ and Supplementary Fig. S5). This orientation of Au tips is different from Au tipped wurtzite nanorods where noncentrosymmetric crystallographic lattice with sulfur-rich facets cause Au tip formation in dumbbells, end-on and angled tip orientations ${ }^{31-33}$.

To understand the alternate attachment and orientational relationship of these Au tips on the dumbbells, we have modeled a truncated octahedron of fcc Au consisting of eight (111) facets and six (200) facets (Fig. 2a) and a cubic PbS nanorod consisting (200) facets (Fig. 2b). If one of these eight $(111)_{\mathrm{Au}}$ facets is attached to the (200) facet of $\mathrm{PbS}$, then the rest (111) planes of $\mathrm{Au}$ will not appear parallel to (200) planes of PbS in TEM micrograph, rather $(111)_{\mathrm{Au}}$ planes will appear oblique to (200) planes of PbS. However, for such an attachment, the (200) planes of Au may appear parallel to the
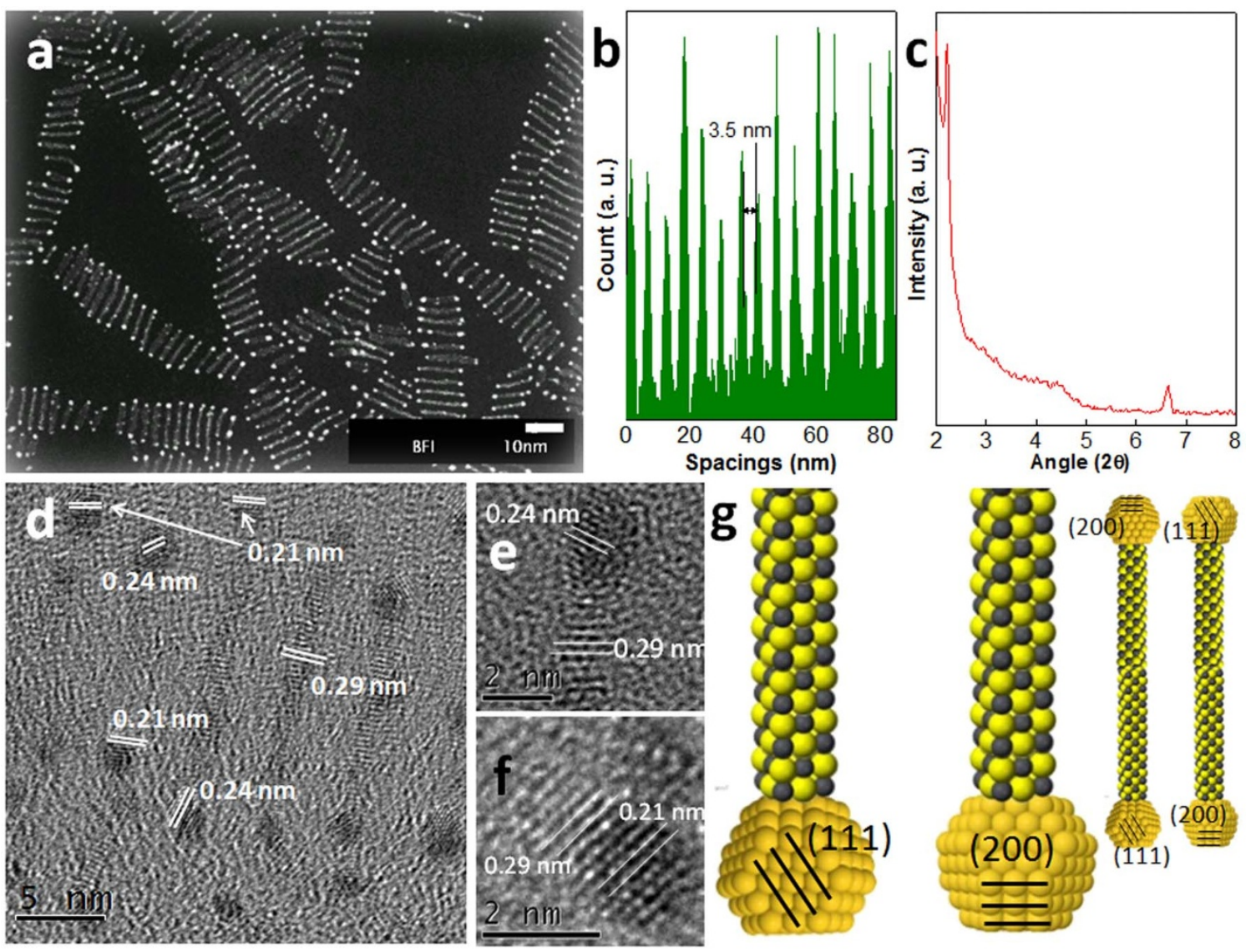

Figure 1 Structural characterizations and self assembly. (a) Dark field TEM image of the dumbbells consists of PbS rods with Au tips. A contrast difference at the tips owing to Au deposition is evidenced, and the average spacing between the bright balls at the tips is $13.8 \pm 2 \mathrm{~nm}$ suggesting $\sim 2 \mathrm{~nm}$ of Au regions at both tips. (b) Statistical spatial distribution of Au-PbS dumbbells showing a lateral separation of $3.5 \mathrm{~nm}$ between the adjacent dumbbells within a single chain. (c) SAXS of dumbbells in toluene suspension. (d) The HRTEM images showing different lattice spacings of PbS and Au tips. Importantly, two different facets of Au have been found at the tips in the alternate arrangements (e) (111) and (f) (200) Au planes at the tips. (g) Schematic of alternate crystallographic orientations of Au tips in the adjacent dumbbells. Inset, adjacent dumbbells with alternate Au facets at the tips. 

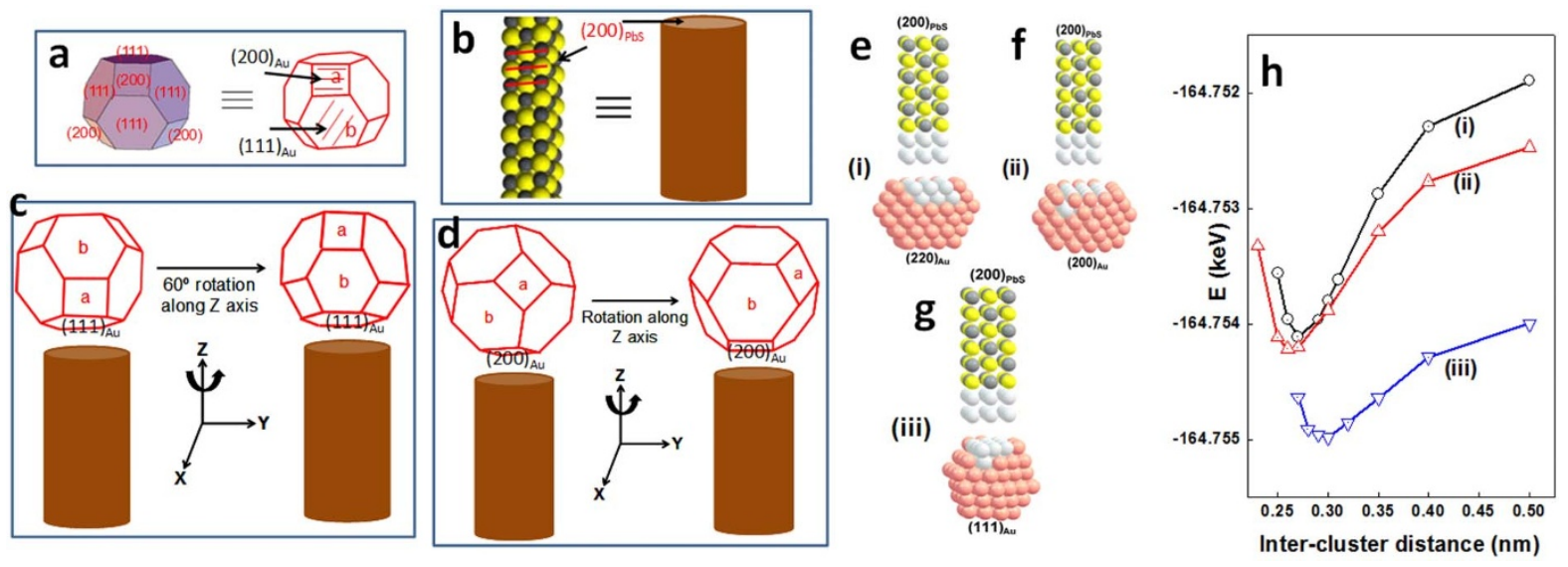

Figure $2 \mid$ Modeling of Au tips attachment on PbS nanorods and DFT calculations of interaction energies of Au tips and PbS nanorod. (a) Model of a truncated octahedron of fcc Au with eight (111) facets and six (200) facets. Equivalent model with (200) Au $_{\text {and }}(111)_{\text {Au }}$ marked by a and b respectively (b) Model of cubic PbS nanorod consisting (200) planes. (c) Model of a Au fcc truncated octahedron at two different orientation keeping the bottom $(111)_{\mathrm{Au}}$ facet fixed along the (200) $)_{\mathrm{PbS}}$ facet. (d) Schematic representation of Au tips attachments on the PbS nanorod through (200) $\mathrm{Au}$ to $(200)_{\mathrm{PbS}}$ planes revealing $(200)_{\mathrm{PbS}}$ cannot be parallel with the $(111)_{\mathrm{Au}}$ or $(200)_{\mathrm{Au}}$ planes. (e), (f) and (g) models of PbS nanorod and Au tips, chosen to estimate the interaction energy between different facets $(220)_{\mathrm{Au}},(200)_{\mathrm{Au}}$ and $(111)_{\mathrm{Au}}$ with the $\mathrm{PbS}(200)$ facets. The pink atoms are Au, yellow atoms are $\mathrm{S}$ and grey atoms are $\mathrm{Pb}$. The white atoms are used for the calculation of interaction energy. (h) Interaction energy as a function of separation when Au clusters are brought closer to PbS clusters for interaction. (i) Interaction between (200) $\mathrm{PbS}$ and (220) $)_{\mathrm{Au}}$. (ii) Interaction between $(200)_{\mathrm{PbS}}$ and (200) $)_{\mathrm{Au}}$. (iii) Interaction between $(200)_{\mathrm{PbS}}$ and $(111)_{\mathrm{Au}}$.

(200) planes of $\mathrm{PbS}$ for a $60^{\circ}$ rotation of the truncated octahedron retaining the bottom $(111)_{\mathrm{Au}}$ facet connected to $(111)_{\mathrm{Pbs}}$. Thus, for the attachment of $(111)_{\mathrm{Au}}$ facet to $(200)_{\mathrm{PbS}}$ facet, one can visualize either oblique (111) $\mathrm{Au}$ (Fig. 2c, $1^{\text {st }}$ combination) or parallel $(200)_{\mathrm{Au}}$ planes (Fig. 2c, $2^{\text {nd }}$ combination). Indeed such an alternate orientation of the Au tips is observed in the HRTEM images. On the other hand, attachment of $(200)_{\mathrm{Au}}$ facet to $(200)_{\mathrm{PbS}}$ is also likely (Fig. 2d). However, for this attachment, neither $(111)_{\mathrm{Au}}$ nor $(200)_{\mathrm{Au}}$ will appear parallel to $(200)_{\mathrm{Pbs}}$ for any degree of rotation of the truncated octahedron.

A means for the existence of alternate orientation of Au tips of the dumbbells is further justified by examining the detail of orientational relationships of the Au tips on the $\mathrm{PbS}$ rods using DFT calculations ${ }^{34}$. The interaction between different facets of $\mathrm{PbS}$ nanorods and $\mathrm{Au}$ tips is estimated using DFT methods performed on atomic clusters representing different facets of Au tips and PbS nanorod. We estimated the possible interactions between (200) facet of PbS nanorod and different $\mathrm{Au}$ facets to evaluate energetically the most favorable Au and PbS interaction. $\mathrm{PbS}$ nanorod is modeled using two layers of $3 \times 3 \mathrm{PbS}$ lattice mimicking $(200)_{\mathrm{PbS}}$ facets, which are probable sites for $\mathrm{Au}$ deposition. Different facets of Au clusters $(3 \times 3$ atoms in two layers) are arranged as $(111)_{\mathrm{Au}},(220)_{\mathrm{Au}}$ and $(200)_{\mathrm{Au}}$ representing the interaction with $(200)_{\mathrm{PbS}}$ nanorod. Different facets of $\mathrm{PbS}$ and Au clusters are gradually brought closer in steps from a large distance and the changes in total energies are calculated. The hybrid functional B3LYP ${ }^{35,36}$ is used along with $6-31++G^{* *}$ basis set for $S$ atoms and Lanl2 $\mathrm{DZ}^{37}$ basis set with effective core potential is used for $\mathrm{Au}$ and $\mathrm{Pb}$ atoms. To validate the results, we have also used $\mathrm{PBE} 1 \mathrm{PBE}^{38,39}$ functional (Supplementary Table S1) using same basis-set. The Au clusters are chosen in such a way that three different facets of Au viz. (i) $(220)_{\mathrm{Au}}$, (ii) $(200)_{\mathrm{Au}}$ and (iii) $(111)_{\mathrm{Au}}$ are exposed to fully interact with $(200)_{\mathrm{PbS}}$ nanorods (Figs. $2 \mathrm{e}-2 \mathrm{~g}$ ).

Each of these Au facets are placed parallel to the $(200)_{\mathrm{Pbs}}$ facet and the corresponding interaction energy is calculated as a function of separation distance between the $\mathrm{Au}$ and $\mathrm{PbS}$ facets (Fig. $2 \mathrm{~h}$ ). This method mimics a qualitative estimate of the energetics in the actual reaction conditions where the direction of approach of Au clusters is random. The energy versus separation curves for all the three combinations show prominent minima corresponding to the optimum bonding distance (Fig. 2h). For example, when the (111) $\mathrm{Au}$ and
$(200)_{\mathrm{PbS}}$ facets [case (iii)] are brought into proximity from a distance of $0.5 \mathrm{~nm}$, a dip in the energy appears at $0.30 \mathrm{~nm}$ corresponding to the most stable adduct. Bonding distances of $0.27 \mathrm{~nm}$ for case (i) and $0.26 \mathrm{~nm}$ for case (ii) combinations are obtained, though the energies for these orientations appear to be higher in comparison to case (iii). Though, the energy for $(220)_{\mathrm{Au}} /(200)_{\mathrm{PbS}}$ and $(200)_{\mathrm{Au}} /(200)_{\mathrm{PbS}} \mathrm{com}-$ binations appear in the same range, however, $(200)_{\mathrm{Au}} /(200)_{\mathrm{PbS}} \mathrm{com}-$ binations always remains to be energetically much stable than $(220)_{\mathrm{Au}} /(200)_{\mathrm{PbS}}$ combinations (Fig. 2h). Additionally, the lattice mismatch for $(111)_{\mathrm{Au}} /(200)_{\mathrm{PbS}}$ sets of planes is $20 \%$ in comparison to $32 \%$ for $(200)_{\mathrm{Au}} /(200)_{\mathrm{PbS}}$ and $51 \%$ of $(220)_{\mathrm{Au}} /(200)_{\mathrm{PbS}}$ suggesting that $(220)_{\mathrm{Au}} /(200)_{\mathrm{PbS}}$ attachment is unlikely. Our DFT calculations also suggest that $(200)_{\mathrm{PbS}} /(111)_{\mathrm{Au}}$ is the most stable configurations followed by $(200)_{\mathrm{Au}} /(200)_{\mathrm{PbS}}$ combinations.

Since the stability of the chains of dumbbells in solution phase is surprising considering the self-assembly formed without the use of direct linkers or templates, we investigate the formation mechanism of chains and the origin for the stability of the assembly in solution. We construct a computationally efficient model to explain the interaction between dumbbells remembering that any model which explains the formation of chains should also explain the origin of alternate orientation of the crystallographic facets of the Au clusters at the tips of the dumbbells. Calculations by us and from the literature on other $1 \mathrm{D}$ nanorods ${ }^{40,41}$ suggest that $\mathrm{PbS}$ nanorods may possess a dipole moment perpendicular to the long ( $\mathrm{z}$ ) axis (Supplementary Fig. S6). The exact value of this moment depends on various factors such as surface reconstruction of the PbS rod. Additionally such surface reconstruction could be enhanced in presence of the spherical Au tips affecting the surface and the epitaxy at the Au-PbS interface. Note that the presence of Au tips only affects the surface reconstruction of the $\mathrm{PbS}$ rod; the reduced Au tips themselves do not contribute to the net dipole moment of the dumbbells due to their symmetrical shape and charge neutrality. We estimated that the dipole moment of the PbS rod may vary from 1 Debye ${ }^{42}$ to $\sim 80$ Debye depending on the surface reconstruction (Supplementary Fig. S6) leading to an attractive interaction between the dumbbells of the order of a few $\mathrm{k}_{\mathrm{B}} \mathrm{T}$ at $5 \mathrm{~nm}$ distance. However, an exact value of the dipole moment of a dumbbell is difficult to assign owing to the intrinsic uncertainty of the structure of the $\mathrm{Au}-\mathrm{PbS}$ interface. Additionally, the interfacial strain originated due to adsorbed TOA 
can also contribute to the net dipole moment. Adsorbed TOA on the surface of the Au tips provides a repulsive interaction, which balances this dipolar attraction between the dumbbells. This repulsive interaction is highly dependent on the orientation of the Au tips of the dumbbells. Recent DFT calculations ${ }^{43}$ reveal that the density of adsorbed amines strongly dependent on the crystallographic facet index. For example, $\{100\}$ facets may have a higher surface density of the ligands than that on the $\{111\}$ facets. Since adsorbed ligands tend to introduce an entropic repulsion between surfaces, the $\{100\}$ facets of the Au tips would like to remain away from each other as far as possible, producing an apparent attractive interaction between the $\{111\}$ surfaces. Further, solvation of the dumbbells within the solvent may also influence the nature of the attractive and repulsive interactions.

Counting the complexities of the different interactions and their relative contribution in our observed assembly, we propose the following "effective" interaction model. We assume that the Au tips are in the form of spheres with an effective radius of $4 \mathrm{~nm}$ accounting the adsorbed TOA layers on actual $\sim 2 \mathrm{~nm}$ Au tips (Figs. 3a and 3b). The interaction between the neighboring Au clusters is therefore driven by the orientation dependent interactions of Au planes covered by TOA, which are modeled by representing the Au clusters as colloidal particles with patchy interactions of the Hamaker type (Fig. $3 \mathrm{c})^{44}$ as implemented in the MD simulation package LAMMPS $^{45}$. Accordingly, each Au cluster therefore consists of one central, large, spherical particle with six small equidistant patches of alternating types on the equator representing the (111) and (200) planes of a truncated octahedron structure (Fig. 3c). We refer the central particle in Fig. $3 \mathrm{c}$ as type-1 particle and the magenta and blue patches on the equator of central particle as type- 2 and type- 3 particles respectively. Thus each dumbbell consists of a rod linking such two patchy colloidal particles (Fig. 3d). The sizes and interaction strengths between type-1, type- 2 and type- 3 particles are chosen so as to reproduce the structures observed in the experiments as closely as possible (Supplementary Table S2). We have calculated the interaction energy $\mathrm{U}\left(\mathbf{r}_{\mathrm{ij}}, \boldsymbol{\omega}_{\mathrm{ij}}\right)$ between two Au clusters of $\mathrm{i}^{\text {th }}$ and $\mathrm{j}^{\text {th }}$ dumbbell separated by the displacement $\mathbf{r}_{\mathbf{i j}}$ and the internal coordinate $\boldsymbol{\omega}_{\mathrm{ij}}$ which measures the relative orientations of the clusters (Fig. $3 \mathrm{~d}$ ). Note that, $\omega_{\mathrm{ii}}$ represents the relative orientation of Au tips of a same dumbbell. Figure $3 \mathrm{e}$ shows the Au tips (patchy particles) of three such dumbbells with relative orientation separated by displacements $\mathbf{r}_{\mathbf{i j}}$ with either $\theta=0$ or $\theta=\pi / 3$ where, $\theta$ (expressed in radians), is the angle between the $x$ axis and the vector $\left(\mathbf{r}_{\mathbf{i j}}\right)$ connecting a pair of the Au tips. A polar plot of the Boltzmann factor $\exp [-\beta U(\theta)]$ as a function of the direction $\theta$ of $\mathbf{r}_{\mathbf{i j}}$ at a fixed value of the magnitude of $\mathbf{r}_{\mathbf{i j}}$ and $\boldsymbol{\omega}_{\mathbf{i j}}=\pi / 3$ is shown in Fig. 3 f. Evidently, the angular dependence of the interaction energy shows a strong, in-plane, 3-fold symmetry in both the cases.

Plots of $U\left(r_{i j}, \omega_{i j}\right)$ for various $\omega_{i j}$ i.e. with different combinations of $\mathrm{Au}$ planes facing each other is shown in Fig. 3g. The interaction energy is calculated as a function of the magnitude of $\mathbf{r}_{\mathbf{i j}}$, for three distinct cases i.e. when (A) two $(111)_{\text {Au }}$ planes face each-other $\left(\omega_{\mathrm{ij}}=\right.$ $\pi / 3$ and $\theta=0),(B)$ one $(111)_{\text {Au }}$ plane faces a $(200)_{\text {Au }}$ plane $\left(\omega_{\mathrm{ij}}=0, \theta\right.$ $=0)$ and $(\mathbf{C})$ when two $(200)_{\text {Au }}$ planes face each-other $\left(\omega_{\mathrm{ij}}=\pi / 3, \theta\right.$ $=\pi / 3, \pi, 5 \pi / 3)$. The interaction for all the three cases have a van der Waals form with attractive minima representing the stability of pairs of Au tips with the specific orientations. Note that, the deepest minimum is obtained for case (A) implying that $(111)_{\mathrm{Au}} \|(111)_{\mathrm{Au}}$ is energetically the most stable combinations followed by (B), $(111)_{\mathrm{Au}} \|(200)_{\mathrm{Au}}$. We find that there are two possible distinct orientational states, namely, the relative orientation $\omega_{\mathrm{ii}}$ between the two $\mathrm{Au}$ tips at either end of a rod may be fixed at 0 or $\pi / 3$. One can verify that these are the only two lower energy configurations allowed by the constraints set by the epitaxy of Au on PbS (Fig. 2h). Note that unlike $\omega_{i j}$, the orientational difference between Au tips of different dumbbells which evolves in time, the quantity $\omega_{\mathrm{ii}}$ is fixed since the $\mathrm{Au}$ tips of a single dumbbell cannot rotate relative to each other. We

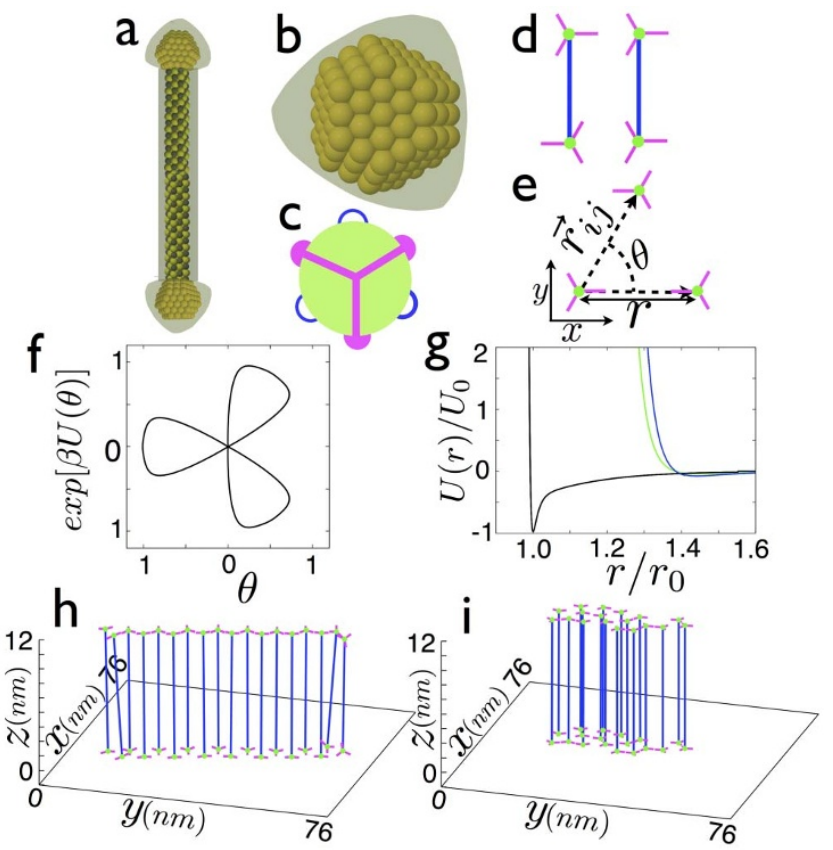

Figure $3 \mid$ Modelling, interaction energy calculations and molecular dynamics simulation. (a) Schematic for Au tipped $\mathrm{PbS}$ dumbbells with TOA surfactant coating (grey shading) and the truncated octahedra (TO) represent the Au clusters on either tips. (b) A single TO Au cluster showing $\mathrm{Au}$ atoms (yellow balls) covered by adsorbed TOA (grey shading).

(c) Representation of the Au cluster in (b) as a patchy spherical colloidal particle (green balls) with patches (magenta and blue balls) along the equator. (d) Representation of the complete dumbbell with an inert rod (blue line) connecting two patchy particles through the centers of magenta patches. The relative orientation $\omega_{\mathrm{ii}}$ between the patches at the two ends of a same dumbbell can have two distinct values viz. $\omega_{\mathrm{ii}}=\pi / 3$ (left rod, configuration (I)) or $\omega_{\mathrm{ii}}=0$ (right rod, configuration (II)). (e) Schematic showing only the Au tips (patchy particles) of three such dumbbells interacting such that either $(111)_{\mathrm{Au}}||(111)_{\mathrm{Au}}(\theta=0)$ or $(111)_{\mathrm{Au}}||(200)_{\mathrm{Au}}$ $(\theta=\pi / 3)$ where $\theta$ is the direction of $r_{i j}$ at a fixed value of the magnitude $r$ of $r_{i j}$. (f) Boltzmann factor $\exp \left(-\beta \mathrm{U}\left(\mathrm{r}_{\mathrm{ij}}, \theta\right)\right), \beta=0.01$ as a function of the direction $\theta$ of $r_{i j}$ at a fixed value of the magnitude $r$ of $r_{i j}$ for two clusters with relative orientation $\omega_{\mathrm{ij}}=\pi / 3$. (g) Interaction energy, $\mathrm{U}\left(\mathrm{r}_{\mathrm{ij}}, \omega_{\mathrm{ij}}\right)$, as a function of $r$ when $(111)_{\mathrm{Au}} /(111)_{\mathrm{Au}}$ planes (black line), $(111)_{\mathrm{Au}} /(200)_{\mathrm{Au}}$ planes (green line) and $(200)_{\mathrm{Au}} /(200)_{\mathrm{Au}}$ (blue line) planes face each-other. Here $r_{0}$ and $U_{0}$ stands for the unit of length and unit of energy respectively. We have chosen $\mathrm{U}_{0} \sim \mathrm{k}_{\mathrm{B}} \mathrm{T}$ and $\mathrm{r}_{0} \sim 4 \mathrm{~nm}$ to correspond to the experimental observation. Final configurations from MD simulation of a chain of dumbbells each containing Au of (h) opposite orientation at both ends $\left(\omega_{\mathrm{ii}}=\pi / 3\right)$ and (i) same orientation $\left(\omega_{\mathrm{ii}}=0\right)$ separated by $12 \mathrm{~nm}$ distance.

constructed two chains each with $\mathrm{N}=18$ dumbbells (I) taking $\omega_{\mathrm{ii}}=$ $\pi / 3$ and (II) with $\omega_{\mathrm{ii}}=0$ for all $\mathrm{i}=1, \mathrm{~N}$. Here after we will define these two chains as configuration (I) and (II) respectively. In the experimental situation, we only observe dumbbells in the configuration (I) i.e. tips of a dumbbell with alternate (111) and (200) combinations, though both configurations should be equally probable. We discuss possible reasons for this anomaly in the later part of the manuscript.

To reproduce the experimental results, we carry out MD simulations using the fast, parallelized package LAMMPS $^{45}$ of both the chains. We equilibrate the system in the constant $\mathrm{N}$, volume $\mathrm{V}$ and temperature $\mathrm{T}$ (NVT-ensemble) for $10^{8} \mathrm{MD}$ steps with an integration time-step $\Delta \mathrm{t}=10^{-4}$ followed by another $10^{8} \mathrm{MD}$ steps in the constant NVE-ensemble, where $\mathrm{E}$ is the total energy of the system. After the system is equilibrated at temperature $\mathrm{T}=0.1$, we collected con- 
figurations for $2 \times 10^{8}$ steps in the NVE ensemble at an interval of 50000 steps. The final orientation for both configurations (I) and (II) systems are shown in Fig. 3h and Fig. 3i respectively. The dumbbells get aligned in a chain-like structure resembling our experimental observations for configuration (I). Note that this alternate orientation of the facets in the Au clusters along the chain seen in the HRTEM images (Fig. 1d) is maintained. For configuration (II), the dumbbells cluster together eventually forming a 2D structure consisting of two layers of graphene-like honeycomb solid structure. Now we need to understand this orientational dependent behavior of dumbbells in configurations (I) and (II). Configuration (I) is incompatible with the formation of a honeycomb solid structure as shown in Fig. 4a since the bonds in one of the layers (consisting of one of the preferred Au tips) is always frustrated.

On the other hand, in case of a 1D chain, this system of dumbbells can introduce a tilt, which removes frustration producing stable chains (Fig. 4b). To further verify this, we simulate a honeycomb lattice of $\mathrm{N}=218$ dumbbells with configuration (II) at a low temperature $\mathrm{T}=0.1$. We perform MD simulations in the NVT-ensemble for $1.5 \times 10^{8}$ steps of size $\Delta t=10^{-4}$ and collect configurations for another $10^{8}$ steps in NVE-ensemble. The final configuration of this simulation is shown in Fig. $4 \mathrm{c}$ which shows that configuration (II) indeed favors the stable honeycomb lattice structure over 1D chains. Finally in Fig. 4d, we have compared the ground state energies as a function of density of four lowest energy structures which may be created using dumbbells. Firstly, a honeycomb lattice of dumbbells with the same orientation of Au tips i.e. $\omega_{\mathrm{ii}}=0$ (configuration II). Second, an array of chains where the distance between neighboring dumbbells (with $\omega_{\mathrm{ii}}=\pi / 3$ ) within the chain was kept fixed so as to obtain the lowest energy (configuration I) and the distance between chains was varied according to density in order to obtain a rectangular lattice of weakly interacting chains. Third, a close packed triangular lattice of dumbbells with $\omega_{\mathrm{ii}}=\pi / 3$ and lastly a honeycomb lattice of dumbbells with $\omega_{\mathrm{ii}}=\pi / 3$. Of these, for $\omega_{\mathrm{ii}}=\pi / 3$ (configuration I), the structure consisting of chains has the lowest energy while the honeycomb structure is extremely unstable because of the effect of frustration as already described. If, on the other hand $\omega_{\mathrm{ii}}=0$ (configuration II), one obtains a two-dimensional honeycomb structure as the stable state which agrees with the results of our simulations. At extremely high densities regardless of whether $\omega_{\mathrm{ii}}=\pi / 3$ or 0 , one obtains a close packed triangular lattice.

A phase-diagram of a similar system in $2 \mathrm{D}$ where only one of the Au tips are considered and out-of-plane fluctuations are neglected is shown in supplementary Fig. S7 in the density-temperature plane for a system of $\mathrm{N}=384$ of patchy colloidal particles. For low densities and low temperatures, this system is in the gas solid coexistence region of the phase diagram, where the chains of dumbbells are formed. At any density, there is a distribution of chain lengths with the average length increasing with the concentration of dumbbells and with decreasing temperature. A crucial quantity in this concern is the critical concentration of dumbbells above which chains become visible. The simulation data (red dots in Supplementary Fig. S7) shows the boundary of the honeycomb solid which forms at higher density. For dumbbells in the configuration (II), the gas solid coexistence region should be dominated by clusters of honeycomb solid instead of chains.

\section{Discussion}

We have demonstrated the formation mechanism of an assembly of $1 \mathrm{D}$ dumbbells in space without linkers based on the relative orientations of the Au clusters at the tip of $\mathrm{PbS}$ rods. The anisotropic van der Waals effective interaction between adjacent Au tips have been modeled to reproduce the dumbbell organization and assembly, which may provide insights into how to control colloidal forces on the macroscale in solution phase. It appears that the concepts developed for the self-assembly of $1 \mathrm{D}$ dumbbells may provide a roadmap for other Au tipped nanorod organizations without template or linkers in solution phase. For example designing a process which selectively separates dumbbells of configurations (I) and (II) may allow to produce a variety of $1 \mathrm{D}, 2 \mathrm{D}$ and $3 \mathrm{D}$ structures with spontaneous selfassembly. However, there remains an open question regarding the stability of configuration (I) as opposed to configuration (II) in the experimental conditions. We believe that other factors such as the epitaxial strain at the $\mathrm{PbS}-\mathrm{Au}$ interface of the dumbbells produced during the Au growth phase may favor configuration (I) with respect to configuration (II) leading to self-assembly in the form of chains. Control of the configuration (I) or (II) in the experimental system may allow obtaining either 1D chains, 2D graphene-like membranes or 3D lattices. It appears that our chains of dumbbells without template or linkers in 3D space offers an added advantage since the welldefined organized assembly is pre-existing in solution phase, where from these assemblies could be anchored on desired locations on solid support for device fabrication. The self assembled dumbbells could be useful for parallel device fabrication where each single dumbbell within a chain will serve as an individual device component. On the other hand, the solution phase assembled dumbbells could be implied for photocatalysis and biomedical applications owing to the existence of ordered metal anchoring points that could bind to a number of substrates with predictable orientation. Our unique assembly may inspire fabricating linker free assemblies using different sizes or types of nanorods by further engineering of
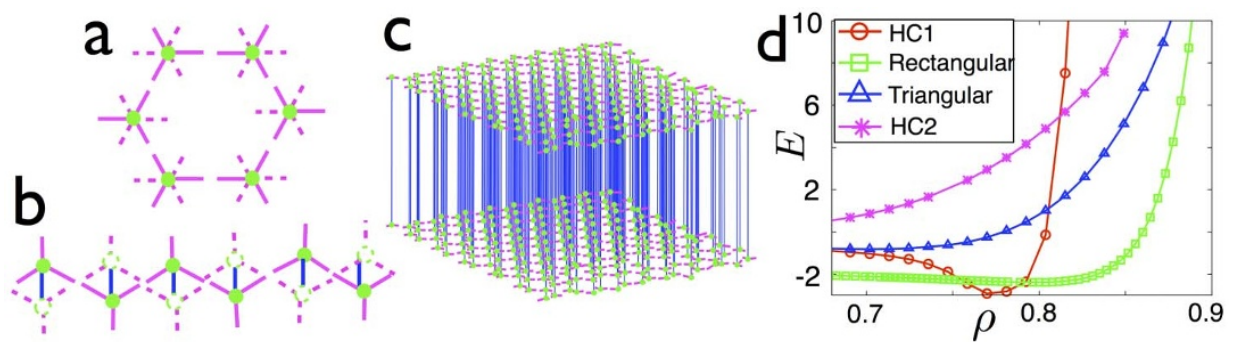

Figure 4 Configurations and ground state energies. (a) Top view of six dumbbells with $\omega_{\mathrm{ii}}=\pi / 3$ showing that the configuration (I) is incompatible with a $2 \mathrm{D}$ honeycomb solid structure. (b) Top view of six dumbbells with $\omega_{\mathrm{ii}}=\pi / 3$ arranged in a chain showing compatibility of configuration (I) in a chain with the dumbbells tilted by an angle of $\tan ^{-1}(\mathrm{D} / \mathrm{L}) \sim 8^{0}$ where $\mathrm{D}$ is the diameter of the Au tips and $\mathrm{L}$ is the length of the PbS rod. (c) Final configuration after $2.5 \times 10^{8} \mathrm{MD}$ steps of a system of 218 dumbbells with same orientation $\left(\omega_{\mathrm{ii}}=0\right)$ contained in a box of dimension $151.2 \mathrm{~nm} \times$ $130.93 \mathrm{~nm} \times 12.0 \mathrm{~nm}$ at $\mathrm{T}=0.1$. (d) Comparison of the scaled energies as a function of density $\rho$ for four different configurations HC1 (red circles) honeycomb structure comprising dumbbells with $\omega_{\mathrm{ii}}=0$; array of weakly interacting chains in a Rectangular lattice (green squares) of dumbbells with $\omega_{\mathrm{ii}}$ $=\pi / 3$ where the interactions within the chain is much larger than between chains; a Triangular lattice with $\omega_{\mathrm{ii}}=\pi / 3$ (blue triangles) and HC2 (magenta stars) a honeycomb structure with $\omega_{\mathrm{ii}}=\pi / 3$ where, the energy had to be divided by 200 in order to plot it on the same graph. Note that for $\omega_{\mathrm{ii}}=\pi / 3$, an array of chains has the lowest energy for all except very high densities. 
colloidal concentration or solvent mediums with different dielectric constants.

\section{Methods}

Materials. Hexadecanol, carbondisulfide, lead nitrate, trioctylamine (TOA) and hydrogen tetrachloroaurate(III) $\left(\mathrm{HAuCl}_{4}\right)$ were purchased from Aldrich and used without further purification. All the solvents used in this work were purged with dry nitrogen for few minutes before starting a reaction.

PbS rod synthesis. $\mathrm{PbS}$ nanorods were synthesized according to the reported $\operatorname{method}^{27,28}$. For the synthesis of $1.7 \mathrm{~nm}$ diameter $\mathrm{PbS}$ rods, lead hexadecylxanthate was prepared using hexadecanol and carbondisulfide and added $(0.065 \mathrm{~g})$ in one shot to $1.6 \mathrm{ml}$ of TOA at $65^{\circ} \mathrm{C}$ with continuous stirring under $\mathrm{N}_{2}$. A grayish-milky color appeared after 5 minutes, and then the temperature was increased to $80^{\circ} \mathrm{C}$. Annealing was carried for 40 minutes at $80^{\circ} \mathrm{C}$. Finally the temperature was reduced to $50^{\circ} \mathrm{C}$ and the rods were collected by washing two times with methanol (centrifuge at $3000 \mathrm{rpm}$ for $3 \mathrm{~min})$ and finally with mixture of dichloromethane and methanol $(5: 40$ by volume) to remove excess TOA.

Au-PbS rod synthesis. The ultra narrow $\mathrm{PbS}$ rods prepared by above mentioned route was dried in ambient conditions and a stock suspension of $1 \mathrm{mg} / \mathrm{ml}$ was prepared in toluene. In a separate test tube, $\mathrm{HAuCl}_{4}$ of $1.2 \mathrm{mM}$ in methanol was prepared in ice bath. Both $\mathrm{PbS}$ suspension in toluene and $\mathrm{HAuCl}_{4}$ in methanol was kept in ice bath before mixing. Then, $3 \mathrm{ml} \mathrm{PbS}$ nanorod suspension was added to the $0.6 \mathrm{ml}$ of $\mathrm{HAuCl}_{4}$ in a separate test tube in ice bath with medium stirring. The stirring was continued for 90 minutes for metal deposition. The bright field and dark field TEM measurements were carried out in JEOL JEM-2100F field emission electron microscope.

1. Whitesides, G. M. \& Grzybowski, B. Self-assembly at all scales. Science 295, 2418-2421 (2002).

2. Murray, C. B., Kagan, C. R. \& Bawendi, M. G. Self-organization of CdSe nanocrystallites into three-dimensional quantum dot superlattices. Science 270, 1335-1338 (1995)

3. Wang, Z. L. Structural analysis of self-assembling nanocrystal superlattices. $A d v$. Mater. 10, 13-30 (1998).

4. Khan, A. H., Maji, S., Chakraborty, S., Manik, N. B. \& Acharya, S Multidimensional self-assembly of peanut shaped $\mathrm{PbS}$ nanostructures. RSC $A d v$. 2, 186-191 (2012).

5. Andres, R. P. et al. Self-assembly of a two-dimensional superlattice of molecularly linked metal clusters. Science 273, 1690-1693 (1996).

6. Steiner, D. et al. Electronic structure and self-assembly of cross-linked semiconductor nanocrystal arrays. Nanotechnology 19, 065201(1-4) (2008).

7. Davis, S. A., Burkett, S. L., Mendelson, N. H. \& Mann, S. Bacterial templating of ordered macrostructures in silica and silica-surfactant mesophases. Nature $\mathbf{3 8 5}$, 420-423 (1997).

8. McMillan, R. A. et al. A self-assembling protein template for constrained synthesis and patterning of nanoparticle arrays. J. Am. Chem. Soc. 127, 2800-2801 (2005).

9. Tang, Z., Kotov, N. A. \& Giersig, M. Spontaneous organization of single CdTe nanoparticles into luminescent nanowires. Science 297, 237-240 (2002).

10. Talapin, D. V., Shevchenko, E. V., Murray, C. B., Titov, A. V. \& Kral, P. Dipoledipole interactions in nanoparticle superlattices. Nano Lett. 7, 1213-1219 (2007).

11. Yang, M., Sun, K. \& Kotov, N. A. Formation and assembly-disassembly processes of $\mathrm{ZnO}$ hexagonal pyramids driven by dipolar and excluded volume interactions. J. Am. Chem. Soc. 132, 1860-1872 (2010).

12. Li, M., Schnablegger, H. \& Mann, S. Coupled synthesis and self-assembly of nanoparticles to give structures with controlled organization. Nature 402, 393-395 (1999).

13. Belman, N. et al. Hierarchical assembly of ultranarrow alkylamine-coated $\mathrm{ZnS}$ nanorods: a synchrotron surface $\mathrm{x}$-ray diffraction study. Nano Lett. 8, 3858-3864 (2008)

14. Auyeung, E. et al. Synthetically programmable nanoparticle superlattices using a hollow three-dimensional spacer approach. Nat. Nanotechnol. 7, 24-28 (2012).

15. Sharma, J. et al. Control of self-assembly of DNA tubules through integration of gold nanoparticles. Science 323, 112-116 (2009).

16. Liu, K., Zhao, N. \& Kumacheva, E. Self-assembly of inorganic nanorods. Chem. Soc. Rev. 40, 656-671 (2011)

17. Salant, A., Amitay-Sadovsky, E. \& Banin, U. Directed self-assembly of gold-tipped CdSe nanorods. J. Am. Chem. Soc. 128, 10006-10007 (2006).

18. Pan, B. et al. DNA-templated ordered array of gold nanorods in one and two dimensions. J. Phys. Chem. C 111, 12572-12576 (2007).

19. Cozzoli, P. D. \& Manna, L. Asymmetric nanoparticles: tips on growing nanocrystals. Nat. Mater. 4, 801-802 (2005).

20. Vinod, T. P., Yang, M., Kim, J. \& Kotov, N. A. Self-guided one-sided metal reduction in Te nanowires leading to Au-Te matchsticks. Langmuir 25, 13545-13550 (2009).

21. Mokari, T., Rothenberg, E., Popov, I., Costi, R. \& Banin, U. Selective growth of metal tips onto semiconductor quantum rods and tetrapods. Science 304, $1787-1790$ (2004)
22. Sheldon, M. T., Trudeau, P.-E., Mokari, T., Wang, L.-W. \& Alivisatos, A. P. Enhanced semiconductor nanocrystal conductance via solution grown contacts. Nano Lett. 9, 3676-3682 (2009).

23. Costi, R., Saunders, A. E., Elmalem, E., Salant, A. \& Banin U. Visible light-induced charge retention and photocatalysis with hybrid CdSe-Au nanodumbbells. Nano Lett. 8, 637-641 (2008)

24. Amirav, L. \& Alivisatos, A. P. Photocatalytic hydrogen production with tunable nanorod heterostructures. J. Phys. Chem. Lett. 1, 1051-1054 (2010).

25. Choi, S.-H. et al. Simple and generalized synthesis of oxide-metal heterostructured nanoparticles and their applications in multimodal biomedical probes. J. Am. Chem. Soc. 130, 15573-15580 (2008).

26. Zhang, Z. \& Glotzer, S. C. Self-assembly of patchy particles. Nano Lett. 4, 1407-1413 (2004).

27. Acharya, S. et al. Ultra narrow $\mathrm{PbS}$ nanorods with intense fluorescence. J. Am. Chem. Soc. 4594-4595 (2008)

28. Khan, A. H. et al. Synthesis and metallic probe induced conductance of Au tipped ultranarrow PbS rods. Chem. Commun. 47, 8421-8423 (2011).

29. Kalsin, A. M. et al. Electrostatic self-assembly of binary nanoparticle crystals with a diamond-like lattice. Science 312, 420-424 (2006).

30. Xia, Y. et al. Self-assembly of self-limiting monodisperse supraparticles from polydisperse nanoparticles. Nat. Nanotechnol. 6, 580-587 (2011).

31. Menagen, G., Macdonald, J. E., Shemesh, Y., Popov, I. \& Banin, U. Au growth on semiconductor nanorods: photoinduced versus thermal growth mechanisms. J. Am. Chem. Soc. 131, 17406-17411 (2009).

32. O’Sullivan, C., Gunning, R. D., Barrett, C. A., Singh, A. \& Ryan, K. M. Size controlled gold tip growth onto II-VI nanorods. J. Mater. Chem. 20, 7875-7880 (2010).

33. Figuerola, A. et al. Epitaxial CdSe-Au nanocrystal heterostructures by thermal annealing. Nano Lett. 10, 3028-3036 (2010).

34. Frisch, M. J. Gaussian03, Revision C.02, Gaussian Inc.: Wallingford, CT (2004)

35. Becke, A. D. Density-functional thermochemistry. III. The role of exact exchange J. Chem. Phys. 98, 5648-5652 (1993).

36. Becke, A. D. Density-functional exchange-energy approximation with correct asymptotic behaviour. Phys. Rev. A 38, 3098-3100 (1998).

37. Wadt, W. R. \& Hay, P. J. Ab initio effective core potentials for molecular calculations. Potentials for main group elements $\mathrm{Na}$ to Bi. J. Chem. Phys. 82, 284-299 (1985).

38. Perdew, J. P., Burke, K. \& Ernzerhof, M. Generalized gradient approximation made simple. Phys. Rev. Lett. 77, 3865-3868 (1996).

39. Adamo, C. \& Barone, V. Toward reliable density functional methods without adjustable parameters: The PBE0 model. J. Chem. Phys. 110, 6158-6170 (1999).

40. Li, L.-S. \& Alivisatos, A. P. Origin of scaling of the permanent dipole moment in CdSe nanorods. Phys. Rev. Lett. 90, 097402 (1-4) (2003).

41. Nann, T. \& Schneider, J. Origin of permanent electric dipole moments in wurtzite nanocrystals. Chem. Phys. Lett. 384, 150-152 (2004).

42. Kundu, S. et al. Ultranarrow PbS nanorod-nematic liquid crystal blend for enhanced electro-optic properties. ACS Appl. Mater. Interfaces 2, 2759-2766 (2010).

43. Halder, A. \& Ravishankar, N. Ultrafine sinfle-crystalline gold nanowire arrays by oriented attachment. Adv. Mater. 19, 1854-1858 (2007).

44. Everaers, R. \& Ejtehadi, M. R. Interaction potentials for soft and hard ellipsoids. Phys. Rev. E 67, 041710 (1-8) (2003).

45. Plimpton, S. Fast parallel algorithms for short-range molecular dynamics. J. Comp. Phys. 117, 1-19 (1995)

\section{Acknowledgments}

This research was partially supported by the DST India and Indo-EU project MONAMI and SFB-TR6 grant. A. H. K. and C. M. acknowledge support from CSIR, India.

\section{Author contributions}

C.M. performed simulation, analyzed data and A.H.K. planned and performed the experiments, analyzed data and co-wrote the manuscript. These authors contributed equally to this work. B.D. carried out the DFT calculations and co-wrote the manuscript. S.A. and S.S. guided the data analysis, co-wrote and edited the paper.

\section{Additional information}

Supplementary information accompanies this paper at http://www.nature.com/ scientificreports

Competing financial interests: The authors declare no competing financial interests.

How to cite this article: Mondal, C., Khan, A.H., Das, B., Acharya, S. \& Sengupta, S. Origin of chains of Au-PbS Nano-dumbbells in space. Sci. Rep. 3, 2612; DOI:10.1038/srep02612 (2013).

(i) This work is licensed under a Creative Commons Attribution-

co ${ }_{\text {BY }}$ NC SA NonCommercial-ShareAlike 3.0 Unported license. To view a copy of this license, visit http://creativecommons.org/licenses/by-nc-sa/3.0 